\title{
Atmospheric Transmittance of AMSU Channels: A Fast Computation Model
}

Tsung-Hua Kuo

Graduate Student, Institute of Space Science, National Central University, Chung-Li, Taiwan 320.,

thlin@csrsr.ncu.edu.tw

Gin-Rong Liu

Professor, Center for Space and Remote Sensing Research, National Central University, Chung-Li, Taiwan 320.

Chen-Gen Huang

Graduate Student, Institute of Atmospheric Science, National Central University, Chung-Li, Taiwan 320.

Tang-Huang Lin

Assistant Professor, Air Force Aeronautical and Technical School, Gang-Shan, Taiwan 820.

Chung-Chih Liu

Assistant Professor, Air Force Aeronautical and Technical School, Gang-Shan, Taiwan 820.

Follow this and additional works at: https://jmstt.ntou.edu.tw/journal

Part of the Engineering Commons

\section{Recommended Citation}

Kuo, Tsung-Hua; Liu, Gin-Rong; Huang, Chen-Gen; Lin, Tang-Huang; and Liu, Chung-Chih (2002) "Atmospheric Transmittance of AMSU Channels: A Fast Computation Model," Journal of Marine Science and Technology: Vol. 10:

Iss. 2, Article 5.

DOI: $10.51400 / 2709-6998.2308$

Available at: https://jmstt.ntou.edu.tw/journal/vol10/iss2/5

This Research Article is brought to you for free and open access by Journal of Marine Science and Technology. It has been accepted for inclusion in Journal of Marine Science and Technology by an authorized editor of Journal of Marine Science and Technology. 


\section{Atmospheric Transmittance of AMSU Channels: A Fast Computation Model}

\section{Acknowledgements}

This study was supported by the National Science Council under the grant No. NSC-89-2625-Z-008-013. We would also like to thank Mr. C. K. Charlie Liang for his assistance in reviewing the English writing. 


\title{
ATMOSPHERIC TRANSMITTANCE OF AMSU CHANNELS: A FAST COMPUTATION MODEL
}

\author{
Tsung-Hua Kuo*, Gin-Rong Liu**, Chen-Gen Huang***, \\ Tang-Huang Lin****, and Chung-Chih Liu*****
}

Key words: AMSU, microwave, atmospheric transmittance.

\begin{abstract}
In this study, the atmospheric transmittance of individual NOAA AMSU microwave channels was computed into a 40-layer atmospheric line-by-line FASCODE radiation model. Long-term sounding data from 1956 to 1991 collected over the Taiwan area was used to construct a 40-layer standard atmospheric model for the island. The simulated transmittance was also used to establish a regression-based fast-algorithm model to compute the atmospheric transmittance of Taiwan and its surrounding areas for various purposes. Despite the fact that the lower atmospheric layers of channels 14-18 indicated larger errors, the overall results revealed that the absolute value difference between the AMSU pass band-averaged transmittances computed through FASCODE and the test model was smaller than 0.01 and 0.001 in the oxygen and water vapor channels, respectively.
\end{abstract}

\section{INTRODUCTION}

Unlike visible and infrared channels, which can only provide information above the cloud top, microwave channels are capable of observing from the surface to the top of the atmosphere. This is made possible by their capability of penetrating cloud layers, and their special interaction with the soil and atmospheric hydrometeors. Consequently, the application of microwave satellite remote sensing has become increasingly crucial both in the analysis of the environment and monitoring of severe weather conditions, especially over the open ocean where traditional observational data are scarce [1-4]. For example, the usage of satellite microwave data can provide more insight in the moni-

Paper Submitted 01/10/02, Accepted 06/13/02. Author for Correspondence: T. Н. Кио.

*Graduate Student, Institute of Space Science, National Central University, Chung-Li, Taiwan 320.E-mail: thlin@csrsr.ncu.edu.tw

**Professor, Center for Space and Remote Sensing Research, National Central University, Chung-Li, Taiwan 320.

*** Graduate Student, Institute of Atmospheric Science, National Central University, Chung-Li, Taiwan 320.

****Assistant Professor, Air Force Aeronautical and Technical School, Gang-Shan, Taiwan 820. toring of severe weather (such as mesoscale convective systems and typhoons) which often occur around oceans, but may cause serious damages when they approach land $[5,6]$. Therefore, the development and application of microwave sensor data has gradually risen to be one of the top researches in the oceanic/atmospheric field. The launch of the Advanced Microwave Sounding Unit (AMSU) mounted on the National Ocean and Atmosphere Administration (NOAA) satellite in 1999, is the latest step toward improving the understanding of the atmospheric temperature/humidity profiles [1, 3, 7]. With its specifically-chosen channels (Table 1), AMSU's multiple-channel sounding data can be used to retrieve the temperature and humidity profiles more accurately, with better vertical and horizontal resolutions than its predecessor [8, 9]. However, atmospheric transmittance values related to the channels are required in advance to perform the retrievals, which play a significant role to efficiently and accurately compute the retrieved results, especially when the so-called physical retrieval methods are involved, which are becoming increasingly popular, as they take into account microwave radiative transfer computations for each retrieval $[10,12]$.

It is possible to generate sets of accurate atmospheric transmittances for such purposes, via line-byline radiation codes, when the computation time is not a concern. However, a faster model would be more useful for the intensity analysis or numerical models of the atmosphere. In order to shorten the computation time, such applications must be computationally efficient so that simplification is necessary. In other words, the line-by-line computation is not a viable method if it is used routinely. Instead, a fast transmittance parametric model is generally employed. To utilize such a method, it is required to compute a set of line-by-line results that cover a wide range of atmospheric conditions. Next, these results are used to produce the coefficients needed within a fast model. For example, McMillin and Fleming $[11,12]$ used a 2 nd-order temperature polynomial to relate the atmospheric transmittance values and the standard atmospheric conditions for constant mix- 
Table 1. Specifications of the AMSU Channels; F1 stands for 57290.334 MHz (from [9])

\begin{tabular}{|c|c|c|c|}
\hline $\begin{array}{l}\text { Channel } \\
\text { number }\end{array}$ & $\begin{array}{l}\text { Passband center } \\
\text { frequencies }(\mathrm{Mhz})\end{array}$ & $\begin{array}{c}\text { Passband } \\
\text { widths (Mhz) }\end{array}$ & $\begin{array}{c}\text { Required } \\
\text { sensitivity (K) }\end{array}$ \\
\hline \multicolumn{4}{|l|}{ AMSU-A1 } \\
\hline 1 & $23800 \pm 72.5$ & $2 \times 125$ & 0.3 \\
\hline 2 & $31400 \pm 50$ & $2 \times 80$ & 0.3 \\
\hline \multicolumn{4}{|l|}{ AMSU-A2 } \\
\hline 3 & $50300 \pm 50$ & $2 \times 80$ & 0.4 \\
\hline 4 & $52800 \pm 105$ & $2 \times 190$ & 0.25 \\
\hline 5 & $53596 \pm 115$ & $2 \times 170$ & 0.25 \\
\hline 6 & $54400 \pm 105$ & $2 \times 190$ & 0.25 \\
\hline 7 & $54940 \pm 105$ & $2 \times 190$ & 0.25 \\
\hline 8 & $55500 \pm 87.5$ & $2 \times 155$ & 0.25 \\
\hline 9 & $57290 \pm 87.5$ & $2 \times 155$ & 0.25 \\
\hline 10 & $57290 \pm 217$ & $2 \times 78$ & 0.4 \\
\hline 11 & $\mathrm{~F} 1 \pm 322 \pm 48$ & $4 \times 36$ & 0.4 \\
\hline 12 & $\mathrm{~F} 1 \pm 322 \pm 22$ & $4 \times 16$ & 0.6 \\
\hline 13 & $\mathrm{~F} 1 \pm 322 \pm 10$ & $4 \times 8$ & 0.8 \\
\hline 14 & $\mathrm{~F} 1 \pm 322 \pm 4.5$ & $4 \times 3$ & 1.2 \\
\hline 15 & $89000 \pm 1000$ & $2 \times 1000$ & 0.5 \\
\hline \multicolumn{4}{|l|}{ AMSU-B } \\
\hline 16 & $89000 \pm 900$ & $2 \times 1000$ & 1.0 \\
\hline 17 & $150000 \pm 900$ & $2 \times 1000$ & 1.0 \\
\hline 18 & $183310 \pm 1000$ & $2 \times 500$ & 1.1 \\
\hline 19 & $183310 \pm 3000$ & $2 \times 1000$ & 1.0 \\
\hline 20 & $183310 \pm 7000$ & $2 \times 2000$ & 1.2 \\
\hline
\end{tabular}

ing ratio gases, Later improvements regarding the variable mixing ratio gases, or consideration of the main gases involved were subsequently proposed [13-15].

In this study, a transmittance computation algorithm based upon a regression computation, with the aim to quickly and accurately complete calculations regarding the application of Taiwan AMSU data is proposed. In addition, the practicality of the faster algorithm was evaluated through comparisons with the original line-by-line transmittance computations.

\section{DATA AND METHODOLOGY}

\section{Data}

In this study, we developed a fast transmittance computation algorithm for the microwave sounding region. The AMSU channels were tested to verify the results. The AMSU, mounted on NOAA-15 and NOAA16 satellites, consists of three parts: AMSU-A1, AMSUA2 and AMSU-B. The first two provide 15 channels $(23.8 \sim 89.0 \mathrm{GHz})$, mainly for retrieving the atmospheric temperature profile from the surface to $2 \mathrm{hPa}$, at a $45 \mathrm{~km}$ ground resolution in-nadir. The latter provides 5 channels $(89.0 \sim 190 \mathrm{GHz})$ mainly for retrieving the water vapor profile from the surface up to $200 \mathrm{hPa}$, at a $15 \mathrm{~km}$

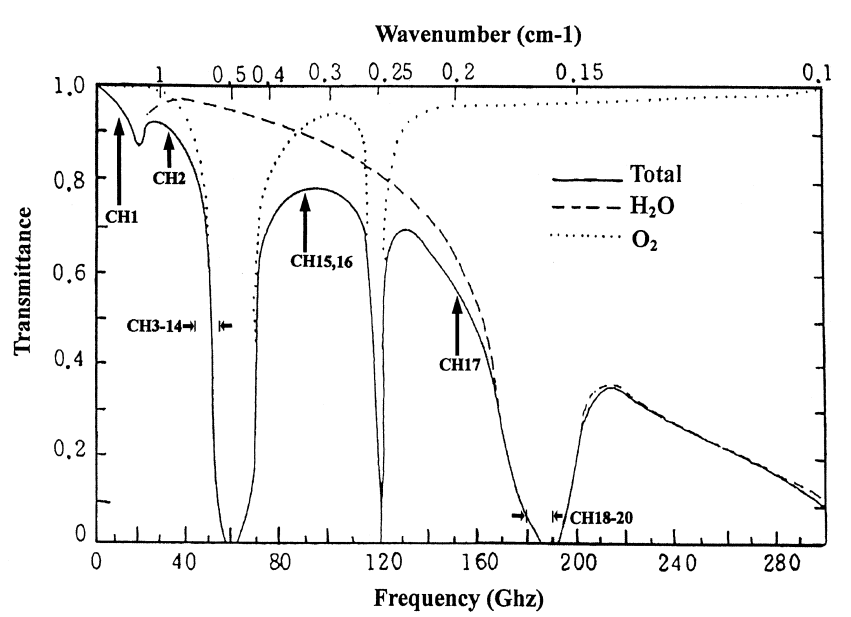

Fig. 1. The transmittances of oxygen, water vapor and the atmosphere in the microwave region which computed by FASCODE3P model and HITRAN92 database [16,17].

resolution [1, 7] (see also Fig. 1).

Radiosonde data collected from 1956 to 1991 around Taiwan were processed by a necessary errorchecking process, and used as the Taiwan standard atmosphere (TSA). In addition, 40 radiosonde data, obtained between 1993 to 1995 were picked to compute the individual transmittance from different layers to the 
top of the atmosphere. The radiation computation code, FASCODE, was employed to perform the computations. To cover different weather conditions, 40 cases were selected, including different months from January to December [16]. Various types of weather phenomena, such as clear or rainy skies, heavy rainfall, typhoons, cold fronts... etc, were selected as input patterns.

\section{Methodology}

To compute the atmospheric transmittance for satellite observation, a line-by-line model gives the most accurate approximation [11],

$$
\begin{aligned}
\tau_{i}(P, \theta)= & \int d v H_{i}(v) \exp \left[-\int_{Z(P)}^{\infty} \sum K_{L}(v, Z)\right. \\
& \left.C_{L}(Z) \rho(Z) d Z \sec \theta\right]
\end{aligned}
$$

where $\tau_{i}$ is the atmospheric transmittance, $P$ is the atmospheric pressure, $\theta$ is the satellite zenith angle, $v$ is the frequency, $H$ is the response function, $i$ is the channel number, $H_{i}(v), Z$ is the height, $K_{L}$ is the absorber coefficient of line $L, C_{L}$ is the mixing ratio of the absorbing gases, and $\rho$ is the air density.

However, a line-by-line model requires a tremendous amount of computational time, and is not appropriate for real time retrievals. Instead, we used FASCODE $3 \mathrm{P}$ to compute the transmittance in a line-by-line mode, under various weather conditions. A quick regression method was found to compute the transmittance for routine operational retrievals.

By employing the most reasonable monochrome assumption for the microwave region, the transmittance can be computed by [16],

$$
\tau_{v}=\tau_{v F} \tau_{v W} \tau_{v O}
$$

where $\tau_{v F}, \tau_{v W}, \tau_{v O}$ are the transmittances of the constant mixing ratio gases, the water vapor, and the other gases, respectively. In general cases, the combined effects should be taken into account. Due to the fact that the absorbing lines for water vapor and oxygen are well separated in the AMSU channels, we can take advantage of the monochrome assumption in our computation. The vertical transmittance between the height of $P_{1}$ to $P_{2}$ was computed by [16],

$$
<\tau\left(P_{1}, P_{2}\right)>=\exp \{-(\alpha+\beta V+\gamma L)\}
$$

where $\langle\tau\rangle$ is the mean transmittance, $\alpha, \beta$ and $\gamma$ are the optical depths of oxygen, water vapor and liquid water, respectively. $V$ and $L$ are the water vapor and liquid water content, respectively.

As mentioned previously, the transmittance of the water vapor and oxygen can be individually computed for AMSU regions. The term $\alpha$ can be expanded with a 3rd-order polynomial,

$$
\alpha=c_{1}+c_{2} q+c_{3} q^{2}+c_{4} q^{3}
$$

and $q=(T c l i /\langle T\rangle)-1$

where $c_{1}, c_{2}, c_{3}, c_{4}$ are the regression coefficients, $\langle>$ is the mean value operator, Tcli is the long-term temperature mean from 1956 to 1991 based upon actual observations in Taiwan, and $\langle T\rangle$ is the mean temperature between the layers $P_{1}$ to $P_{2}$. Similarly, $\beta$ was expanded by the same process, as well as the water vapor pressure, which is combined together under the following form,

$$
\beta=d_{1}+d_{2} q+d_{3} q^{2}+d_{4} q^{3}+d_{5}\left\langle P_{\mathrm{H}_{2} \mathrm{O}}\right\rangle
$$

where $d_{1}$ to $d_{5}$ are the regression coefficients, and $\left\langle P_{\mathrm{H}_{2} \mathrm{O}}\right\rangle$ is the mean pressure between the layers $P_{1}$ to $P_{2}$.

Also, the $\alpha$ and $\beta$ values both can be computed by a radiation model simulation based upon the following,

$$
\alpha(\text { or } \beta)=\ln \left(\left\langle\tau_{o}\left(0, P_{1}\right)\right\rangle /\left\langle\tau_{o}\left(0, P_{2}\right)>\right)\right.
$$

The $\alpha$ and $\beta$ values were computed by the FASCODE model, with a 40-layer paralleled-stratified atmosphere (Table 2). The 40 previously mentioned sounding data sets from the AMSU's 20 channels were the input data where we established their regression relationships with the temperature and humidity values. Finally, a fast transmittance computation algorithm for the atmosphere of Taiwan and its neighboring areas was obtained (Fig. 2).

\section{SIMULATION RESULTS AND APPLICATION}

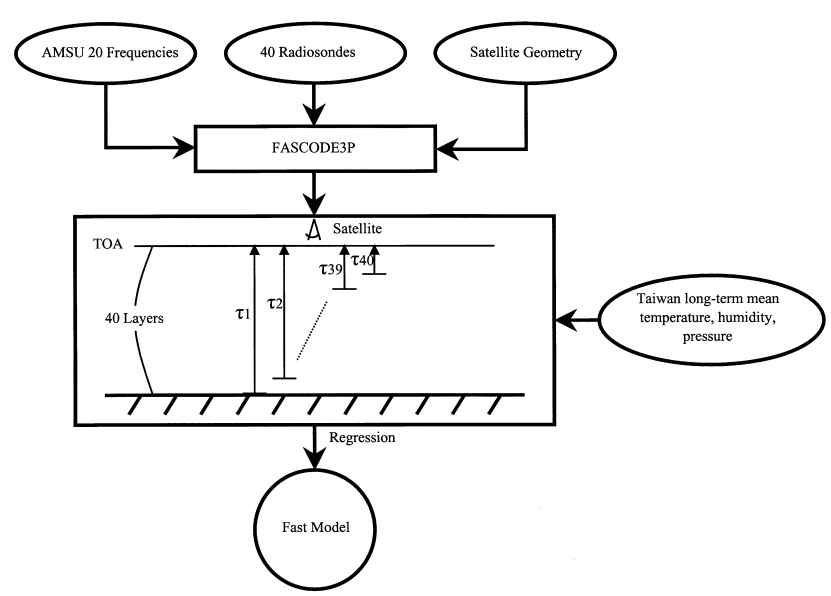

Fig. 2. Flowchart of the fast model. 
Basically, the computation results from a line-byline model are capable of providing extremely accurate atmospheric transmittance values, where its result may be used for verification purposes. However, the method is generally considered too slow for such physical retrieval methods. Therefore, in order to be more useful, a parametric-simplification fast model is constructed. The transmittance computation result produced from the fast model is then compared with the line-by-line model to evaluate its accuracy.

After establishing the fast regression algorithm, we can estimate the vertical atmospheric transmittance related to the AMSU's channels, between an arbitrary pressure layer with the top of the atmosphere (satellite). The fast model is used to compute the atmospheric transmittance at 20 AMSU channels from each of the forty pressure levels to the top of the atmosphere $(0.1$ to $1000 \mathrm{hPa}$, see also Fig. 2 and Table 2). In this study, the verification process is mainly done by comparing the different responses of oxygen and water vapor effects within the entire vertical profiles (e.g. from the surface to the top of the atmosphere in a nadir viewing angle). We compared the transmittance computed by our fast model and by the FASCODE line-by-line mode to evaluate our model's accuracy. For each AMSU channel, we computed separately the oxygen and water vapor transmittance of the atmosphere for the 40 radiosondes selected under different weather conditions from 1993 to 1995 by our fast model and the FASCODE3P model. Due to the fact that their consistency is what concerns us the most, the absolute transmittance accuracy is of little importance. Therefore, the correlation relationship is instead used to evaluate our fast model's practicality. Generally speaking, the results revealed that the oxygen transmittance of the AMSU channels 10 to 14 had higher errors near the surface. However, such errors would not significantly influence the various retrieval applications, because the weighting functions of these channels were above $500 \mathrm{mb}$.

For other channels and other layers, the errors were all smaller than 0.01. For the water vapor channel, the errors were even smaller than 0.001. The transmittances of oxygen and water vapor, from the AMSU channels, are shown in Figs. 3 and 4, respectively. The results indicate that the accuracy is satisfactory. Slightly higher errors exist for channels 14 to 18 , especially in

Table 2. The 40-layer standard atmosphere used in this study; units are in hPa

\begin{tabular}{cccccccccc}
\hline Level & $\mathrm{P}$ & Level & $\mathrm{P}$ & Level & $\mathrm{P}$ & Level & $\mathrm{P}$ & Level & $\mathrm{P}$ \\
\hline 1 & 0.1 & 9 & 5.0 & 17 & 60 & 25 & 250 & 33 & 620 \\
2 & 0.2 & 10 & 7.0 & 18 & 70 & 26 & 300 & 34 & 670 \\
3 & 0.5 & 11 & 10 & 19 & 85 & 27 & 350 & 35 & 700 \\
4 & 1.0 & 12 & 15 & 20 & 100 & 28 & 400 & 36 & 780 \\
5 & 1.5 & 13 & 20 & 21 & 115 & 29 & 430 & 37 & 850 \\
6 & 2.0 & 14 & 25 & 22 & 135 & 30 & 475 & 38 & 920 \\
7 & 3.0 & 15 & 30 & 23 & 150 & 31 & 500 & 39 & 950 \\
8 & 4.0 & 16 & 50 & 24 & 200 & 32 & 570 & 40 & 1000 \\
\hline
\end{tabular}
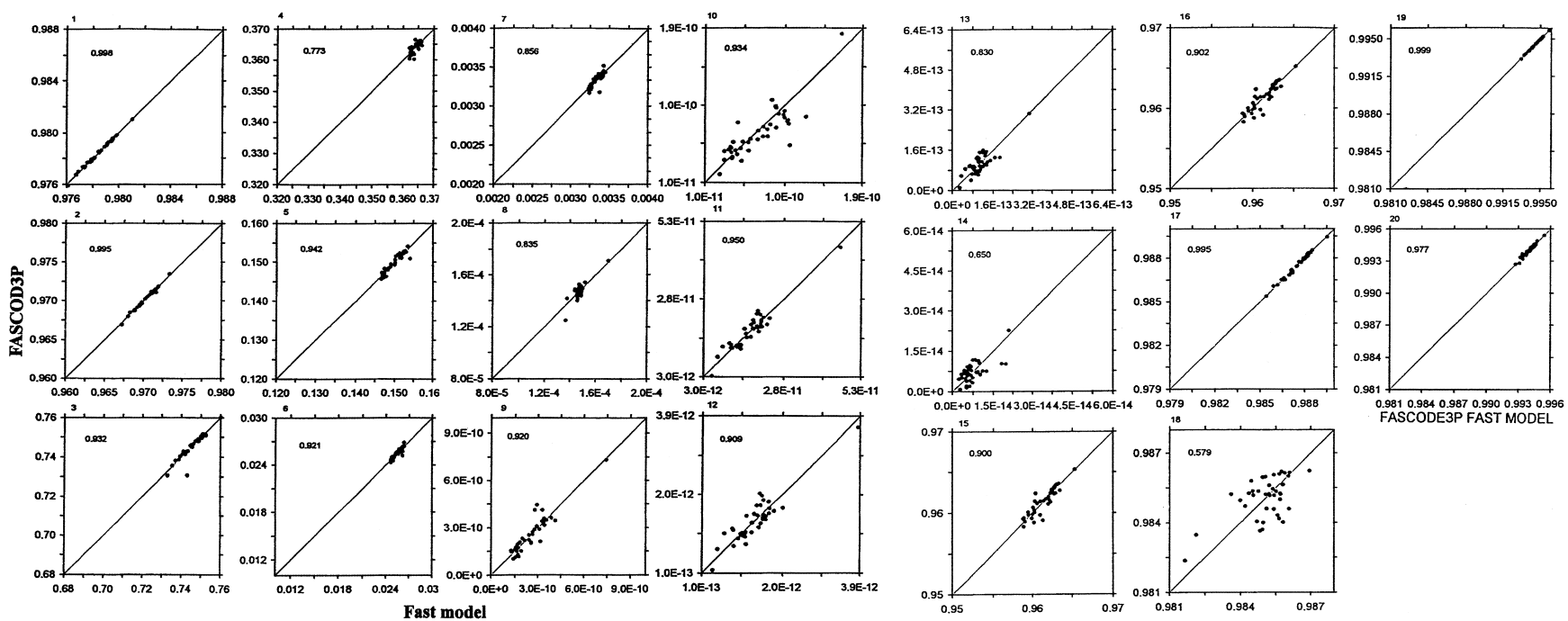

FASCODE3P FAST MODEL

Fig. 3. A comparison of the transmittances for the oxygen component calculated by our fast regression model (x-axis) and FASCODE3P model (y-axis), for the AMSU's channels. The numbers appearing in the upper left corner of each channel are their correlation coefficients. 


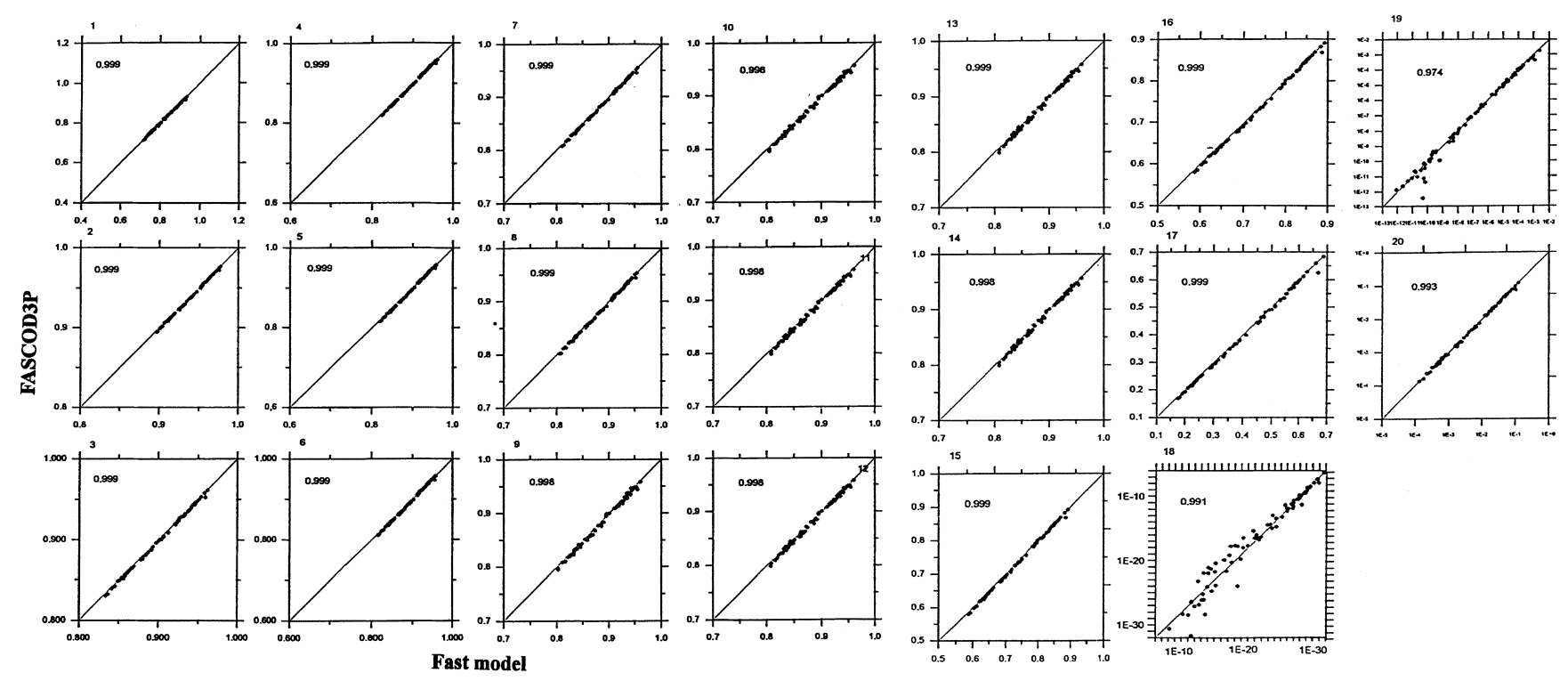

Fig. 4. Same as Fig. 3, except for the water vapor component.

Table 3. Comparison of the oxygen and water vapor transmittance computed by our fast model and the fast model used Rosenkranz algorithm [17]

\begin{tabular}{|c|c|c|c|c|}
\hline \multirow{2}{*}{$\begin{array}{l}\text { AMSU } \\
\text { channel }\end{array}$} & \multicolumn{2}{|c|}{ Our fast model } & \multicolumn{2}{|c|}{ Rosenkranz fast model } \\
\hline & oxygen & water vapor & oxygen & water vapor \\
\hline 1 & 0.998 & 0.999 & 0.913 & 0.993 \\
\hline 2 & 0.995 & 0.999 & 0.995 & 0.994 \\
\hline 3 & 0.932 & 0.999 & 0.932 & 0.996 \\
\hline 4 & 0.773 & 0.999 & 0.444 & 0.996 \\
\hline 5 & 0.942 & 0.999 & 0.748 & 0.996 \\
\hline 6 & 0.921 & 0.999 & 0.700 & 0.996 \\
\hline 7 & 0.856 & 0.999 & 0.566 & 0.996 \\
\hline 8 & 0.835 & 0.999 & 0.247 & 0.996 \\
\hline 9 & 0.920 & 0.998 & 0.862 & 0.994 \\
\hline 10 & 0.934 & 0.998 & 0.854 & 0.994 \\
\hline 11 & 0.950 & 0.998 & 0.834 & 0.834 \\
\hline 12 & 0.909 & 0.998 & 0.782 & 0.782 \\
\hline 13 & 0.830 & 0.999 & 0.660 & 0.994 \\
\hline 14 & 0.650 & 0.998 & 0.519 & 0.994 \\
\hline 15 & 0.900 & 0.999 & 0.820 & 0.996 \\
\hline 16 & 0.902 & 0.999 & 0.823 & 0.996 \\
\hline 17 & 0.995 & 0.999 & 0.920 & 0.996 \\
\hline 18 & 0.579 & 0.991 & 0.622 & 0.999 \\
\hline 19 & 0.999 & 0.974 & 0.920 & 0.994 \\
\hline 20 & 0.977 & 0.993 & 0.885 & 0.994 \\
\hline
\end{tabular}

the upper layers, probably caused by the effect of the earth's magnetic field, which we did not take into account in our model. For the water vapor influence, there was a rather high correlation $(\cong 1.0)$. In addition, we also used the correlation coefficients to compare the transmittance computed from our fast model to one derived from the fast model constructed from the algo- rithm purposed by Rosenkranz [16]. The results showed our model had a higher level of accuracy, especially in the transmittance computation for the oxygen absorbing effect. In addition, a more apparent difference can be seen for the water vapor transmittance in AMSU channels 11 and 12 (see also Table 3). Overall, the fast model developed in this study reveals a higher precision 
than other relevant models, and it also demonstrates its applicability.

\section{CONCLUDING REMARKS}

Basically, the McMillin-Fleming approach was employed to calculate faster, but at the same time, retain its accuracy. In our approximation, the accuracy of the line-by-line computation was kept, but was more efficiently computed. Generally speaking, this is useful for the processing of relevant remotely sensed data in the microwave region, especially for AMSU temperature/ humidity profile retrieving. However, more efforts to improve the transmittance accuracy is encouraged. For example, the addition of the earth's magnetic field effects may likely increase the accuracy for the upper atmospheric layers, making the model more applicable for most tropospheric sounding research, even for stratospheric layers.

In addition, the correctness of this "faster model" is closely dependent on the accuracy of the line-by-line radiation code and absorbing line database, which the fast regression approximation relies on. Although FASCODE can provide enough reliability for most applications, more investigations and further verifications should be conducted. Surely, more observational data is still required to determine the complex relationships of the transmittance with oxygen and water vapor, or other even smaller mixing ratio absorbing gases.

\section{ACKNOWLEDGMENTS}

This study was supported by the National Science Council under the grant No. NSC-89-2625-Z-008-013. We would also like to thank Mr. C. K. Charlie Liang for his assistance in reviewing the English writing.

\section{REFERENCES}

1. Diak, G.R., Kim, D., Whipple, M.S. and Wu. X.,"Preparing for the AMSU," Bull. Am. Meteor. Soc., Vol. 73, No 12, pp. 1971-1984 (1992).

2. Eyre, J.R., "The information content of data from satellite sounding systems: Asimulation stuty," $Q$. J. R. Meteor. Soc., Vol. 116, pp. 401-434 (1989).

3. Muller, B.M., Fullberg, H.E. and Xiang. X., "Simulations of the effect of water vapor, cloud liquid water, and ice on AMSU moisture channel brightness tempertures, "Appl. Meteorl., Vol. 33, pp. 1133-1154 (1994).

4. Smith, W.L., and Achtor. T.H., "The use of visible data in VAS temperature soundings," Appl. Opt., Vol. 27, No. 12, pp. 1309-1315 (1988).

5. Liu, G.R., T.H. Kuo, P.Y. Leu, Lin, T.H. and Liu. C.C., "An estimation of typhoon intensity and the prediction of its track by using MSU data," TAO, Vol. 12, No. 4, pp. 615-634.

6. Liu, G.R., Liu, C.C. and Kuo. T.H., "A contrast and comparison of near-sea surface air temperature/humidity form GMS and SSM/I data with an Improved Alogrithm," IEEE Trans. Geosci. Rem. Sens., Vol. 39, No. 10, pp. 2148-2157 (2001).

7. Mo, T., "Prelaunch calibration of the advanced Microwave Sounding Unit-A for NOAA-K," IEEE. Trans. Microw. Theor. Tech. Vol. 44, No. 8, pp. 1460-1469 (1996).

8. Hewison, T. J. and R. Saunders., "Measurements of the AMSU-B antenna pattern," IEEE Trans. Geosci. Rem. Sens., Vol. 34, No. 2., pp. 405-412 (1996).

9. Saunders, R.W., Hewison, T.J., Stringer, S.J. and Atkinson. N.C., "The radiometric characterization of AMSU-B," IEEE Trans. Microw. Theor. Tech., Vol. 43, No. 4, pp. 760-771 (1995).

10. Eyre, J.R. and Woolf. H.M., "Transmittance of atmospheric gases in the microwave region: a fast model," Appl. Opt., Vol. 27, No. 15, pp. $3244-3249$ (1988).

11. McMillin, L.M. and Fleming. H.E., "Atmospheric transmittance of an absorbing gas: A computationally fast and accurate transmittance model for absorbing gases with constant mixing ratio in inhomogeneous atmospheres," Appl. Opt., Vol. 15, No. 2, pp. 358-363 (1976).

12. Fleming, H.E. and McMillin., L.M., "Atmospheric transmittance of an absorbing gas 2.: A computationally fast and accurate transmittance model for slant paths at different zenith angles," Appl. Opt., Vol. 16, No 5, pp. 1366-1370 (1977).

13. McMillin, L.M., Fleming, H.E. and Hill. M.L., "Atmospheric transmittance of an absorbing gas 3: A computationally fast and accurate transmittance model for absorbing gases with accurate transmittance mixing ratio," Appl. Opt., Vol. 18, No. 10, pp. 1600-1606 (1977).

14. McMillin, L.M., Crone, L.J., Goldberg, M.D. and Kleespies. T.J., "Atmospheric transmittance of an absorbing gas 4.: A computationally fast and accurate transmittance model for absorbing gases with fixed and with variable mixing ratios at variable viewing angles," Appl. Opt., Vol. 34, No. 27, pp. 6269-6274 (1995).

15. McMillin, L.M., Crone, L.J. and Kieespies. T.J., Atmospheric transmittance of an absorbing gas 5.: Improvements to the OPTRAN approach," Appl. Opt., Vol. 34, No. 36, pp. 8396-8399 (1995).

16. Rosenkranz, P.W., "A rapid atmospheric transmittance algorithm for microwave sounding channels," IEEE Trans. Geosci. Rem. Sens., Vol. 33, No. 5, pp. 1135-1140 (1995).

17. Susskind, J., Rosenfield, J. and Reuter. D., “An accurate transfer model for use in the direct physical inversion of HIRS and MSU temperature sounding data," Geophys. Res., Vol. 88, No. C13, pp. 8550-8568 (1983). 